Research Journal of Applied Sciences 13 (11): 681-685, 2018

ISSN: $1815-932 \mathrm{X}$

(C) Medwell Journals, 2018

\title{
Development and Study the Effect of Sintering Temperature on Structural of Cadmium Ferrite Nanoparticles prepared by Ceramic Method
}

\author{
${ }^{1}$ Zena Mohammed Ali Abbas, ${ }^{2}$ Zaid Abdulhadi Abed and ${ }^{3}$ Nada Ismael Ibrahim \\ Department of Physics, College of Science, University of Diyala, Baqubah, Iraq
}

\begin{abstract}
In the present research cadmium ferrite was prepared by standard ceramic method and characterized by XRD and SEM techniques. The X-ray analysis confirms the formation of single phase cubic spinel structure. The crystallite size calculations using XRD Scherer's formula confirms that crystallite size of the cadmium ferrite nanoparticles was 23.1 and $34.75 \mathrm{~nm}$ at a sintering temperatures of 800 and $1000^{\circ} \mathrm{C}$, respectively. The crystallite size of the samples increases with increase in sintering temperature. From the micrograph we can observe the formation of soft agglomerates with irregular morphology constituted the quite fine particles. Since, the ceramic method involves sintering of the stoichiometric mixtures at high temperatures.
\end{abstract}

$\underline{\text { Key words: Cadmium ferrite, the X-ray density, SEM, standard ceramic method, stoichiometric, micrograph }}$

\section{INTRODUCTION}

Ferrites are ceramic, homogeneous materials composed of various oxides with iron oxide as their main constituent. Ferrites can have several distinct crystal structures. However, for this brochur, we are only concerned with the magnetitally soft ferrites which have a cubic crystal structure (Suite, 1998). Ferrites are usually non-conductive ferrimagnetic ceramic compounds derived from iron oxides such as hematite $\left(\mathrm{Fe}_{2} \mathrm{O}_{3}\right)$ or magnetite $\left(\mathrm{Fe}_{3} \mathrm{O}_{4}\right)$ as well as oxides of other metals and are like most other ceramics, hard and brittle. In terms of their magnetic properties, the different ferrites are often classified as "Soft" or "Hard" which refers to their low or high magnetic coercivity (Carter and Norton, 2007). Ceramic method is economical for the bulk synthesis of ferrites but undesired non-uniform particles are formed due to aggregation of nanoparticles and phase purity is not completely achieved. Highenergy milling can reduce the crystallite size but this physical process not only requires greater energy consumption but also easily induces structural disorder in the crystallites (Pradhan et al., 2005).

The properties of nanoferrites are influenced by the composition and microstructure which are sensitive to the preparation methodology used in the synthesis and to the sintering conditions. Ferrite nanoparticles are usually prepared by various physical and chemical methods like high energy milling, plasma deposition, inert gas condensation, citrate precursor technique, reverse micelle technique, microemulsion, hydrothermal reaction, polymer pyrolysis, sol-gel technique, chemical coprecipitation, etc., (Chatterjee et al., 1993; Mishra et al., 2006). The solid-solid reaction between $\mathrm{CdO}$ and $\mathrm{Fe}_{2} \mathrm{O}_{3}$ takes place at a temperature about $6000^{\circ} \mathrm{C}$ gives $\mathrm{CdFe}_{2} \mathrm{O}_{4}$ of moderate degree of crystallinity (Mostafa et al., 2006). Cadmium ferrite has a normal spinel structure (Yokoyama, 1996). The structural, electrical, magnetic and electrical switching properties of cadmium ferrite have been already studied (Gadkari et al., 2009; Vaingankar et al., 1997). At the higher sintering temperature, density gets decreased due to increase in intra-granular porosity resulting from the discontinuous in the grain growth. Electrical switching phenomenon was first reported by Yamashiro (1973).

In the present communication, we report the effect of sintering temperature on structural and electrical switching properties of cadmium ferrite. In industrial electronics ferrites are play an important role as material. In this report, we have presented the combustion synthesis technique for synthesizing cadmium ferrite and their subsequent characterization by XRD, ESM techniques.

\section{MATERIALS AND METHODS}

Ferrite sample preparation: The $\mathrm{CdFe}_{2} \mathrm{O}_{3}$ was prepared by standard ceramic method. The powder of $0.4 \% \mathrm{CdO}$ and $0.6 \%$ ferrous oxide are mixed to obtain a uniform distribution of the components using a variable speed electric mixer for $2 \mathrm{~h}$ for the purpose of obtaining a homogeneous mixture and the nonagglomerated mixtures are then dried in an oven at $80^{\circ} \mathrm{C}$ for $3 \mathrm{~h}$. A mold

Corresponding Author: Zena Mohammed Ali Abbas, Department of Physics, College of Science, University of Diyala, Baqubah, Iraq 
isdesigned for the manufacture of samples in the form of pellet in diameter $(9 \mathrm{~mm})$ and thickness $(5 \mathrm{~mm})$ and the weight of the sample is $(1.5 \mathrm{~g})$. the powder was pressed under hydraulic press with the pressure is (500-700 psi) $5 \mathrm{~min}$ to form $9 \mathrm{~mm}$ diameter pellet. Finally, the pellets were sintered for $4 \mathrm{~h}$ at two sintering temperatures $(800$, $1000^{\circ} \mathrm{C}$ ). The cooling and heating rate of the furnace is at $80^{\circ} \mathrm{C} / \mathrm{h}$. The physical density of the sample was investigated by the Archimedes principle. The X-ray diffraction pattern were recorded using XRD-6000 with $\mathrm{CuK} \alpha\left(\lambda=1.5406 \mathrm{~A}^{\circ}\right)$ that have an accelerating voltage of $220 / 50 \mathrm{~Hz}$ which is produced by Shimadzu company and the scanning electron microscope used in imaging the nanoparticles was a VEGA//EasyProbe which is a favorable combination of a scanning electron microscope and a fully integrated energy dispersive X-ray microanalyser produced by TESCAN, s.r.o., Libusina trida.

\section{RESULTS AND DISCUSSION}

\section{XRD pattern of cadmium ferrite}

Sample sintered at $\left(\mathbf{8 0 0}^{\circ} \mathrm{C}\right)$ : The $\mathrm{X}$-ray diffraction patterns of cadmium ferrite sintered at $\left(800^{\circ} \mathrm{C}\right)$ under investigation are presented in Fig. 1. The XRD confirms the formation of single phase cubic spinel structure. The presence of (311), (422), (220), (511) and (440) planes were observed. The X-diffraction patterns agree with JCPDS card number -22.1063 .

The average crystallite size was calculated using the Scherrer's formula (Nath et al., 2007; Hall et al., 2000) from the broadening of XRD peak corresponding to most intense (311) peak of the XRD pattern. The detailed analysis of the XRD and the assignments of various reflections peaks are given in Table 1 .
Particle size calculation from $\mathrm{X}$-ray diffraction: Average particle size has been estimated by using Scherrer equation:

$$
\begin{aligned}
& \mathrm{D}=0.9 \lambda / \beta \cos \theta \\
& \mathrm{D}=23.1 \mathrm{~nm}
\end{aligned}
$$

The Lattice constant ' $a$ ' was calculated using the relation: Lattice constant of sintered cadmium ferrites under investigation was calculated using the Bragg's equation (Cullity and Stock, 2001):

$$
\begin{aligned}
& \mathrm{a}=\mathrm{d}_{\mathrm{hkl}} \sqrt{\mathrm{h}^{2}+\mathrm{k}^{2}+\mathrm{1}^{2}} \\
& \mathrm{a}=8.55 \mathrm{~nm}
\end{aligned}
$$

where, $\mathrm{h}, \mathrm{k}, 1$ are the Miller indices of the crystal planes and $d_{h k l}$ is the inter planer spacing. The lattice constant obtained from XRD data is in reported range.

\section{The $X$-ray density $\left(d_{x}\right)$ was calculated using the relation:}

Where:

$$
\begin{aligned}
\mathrm{d}_{\mathrm{x}} & =\frac{8 \mathrm{M}}{\mathrm{Na}^{3}} \\
\mathrm{~d}_{\mathrm{z}} & =6.0 \mathrm{~g} / \mathrm{cm}^{3}
\end{aligned}
$$

$\mathrm{M}=$ Molecular weight

$\mathrm{N}=$ Avogadro's number

$\mathrm{a}=$ The Lattice constant

The X-ray density is found to be of the order of $5.42 \mathrm{~g} / \mathrm{cm}^{3}$. The bulk density of cobalt ferrite nano particles

\begin{tabular}{lccc}
\multicolumn{4}{l}{ Table 1: Strongest three peaks of Fig.1 } \\
\hline Peak No. & $2 \theta\left(^{\circ}\right)$ & $\mathrm{d}\left(\mathrm{A}^{\circ}\right)$ & FWHM $\left(^{\circ}\right)$ \\
\hline 4 & 34.7518 & 2.57937 & 0.34050 \\
2 & 29.5875 & 3.01676 & 0.32070 \\
10 & 55.3419 & 1.65872 & 0.26530 \\
\hline
\end{tabular}

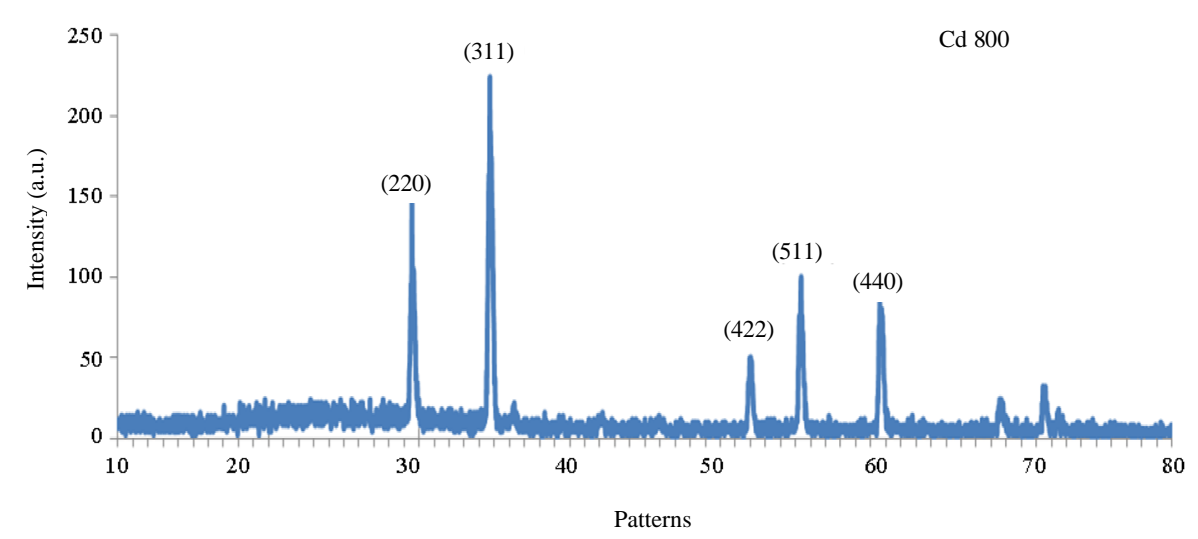

Fig. 1: XRD pattern of cadmium ferrite sintered at $800^{\circ} \mathrm{C}$ 


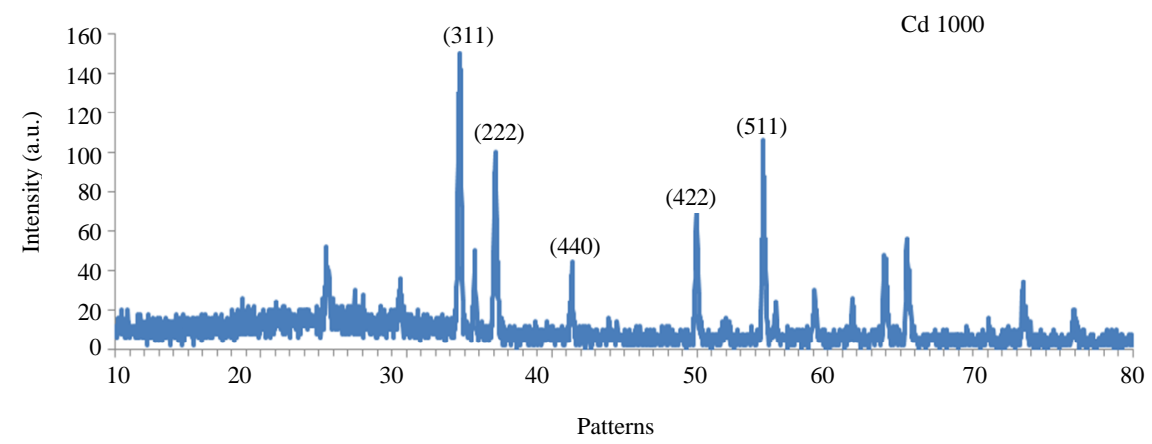

Fig. 2: XRD pattern of cadmium ferrite sintered at $1000^{\circ} \mathrm{C}$

\begin{tabular}{l}
\multicolumn{4}{l}{ Table 2: Strongest three peaks of Fig. 2} \\
\begin{tabular}{lccc}
\hline Peak No. & $2 \theta\left(^{\circ}\right)$ & $\mathrm{d}\left(\mathrm{A}^{\circ}\right)$ & FWHM $\left(^{\circ}\right)$ \\
\hline 26 & 33.6792 & 2.65902 & 0.24000 \\
43 & 54.5750 & 1.68021 & 0.19460 \\
29 & 36.1354 & 2.48371 & 0.22570 \\
\hline
\end{tabular}
\end{tabular}

was measured from the Archimedes principle. The value of bulk density is of the order of $3 \mathrm{~g} / \mathrm{cm}^{3}$. The apparent density calculated by Archimedes method (Actual Density (D)) and the calculation of porosity using the equation (Ahmed et al., 2008):

$$
\begin{aligned}
& \text { Porosity }=[1-(\mathrm{D} / \mathrm{DX})]^{*} 100 \% \\
& \text { Porosity } \%=47.5 \%
\end{aligned}
$$

Sample sintered at $\left(\mathbf{1 0 0 0}^{\circ} \mathrm{C}\right)$ : The X-ray diffraction patterns of cadmium ferrite sintered at $\left(1000^{\circ} \mathrm{C}\right)$ under investigation are presented in Fig. 1. The XRD confirms the formation of single phase cubic spinel structure. The presence of (311), (422), (222) and (400) planes were observed. The X-diffraction patterns agree with JCPDS card number -22.1063 .

Figure 2 shows the X-ray diffraction pattern of cadmium ferrite nano-particles. A careful examination of XRD pattern reveals the appearance of slightly broader peaks signifying the low crystallite size of the prepared samples. All the peak belongs to cubic spinel structure and the analysis of XRD pattern prove the formation of single phase samples. The average crystallite size was calculated using the Scherrer's formula (Nath et al., 2007; Hall et al., 2000) from the broadening of XRD peak corresponding to most intense (311) peak of the XRD pattern. The detailed analysis of the XRD and the assignments of various reflections peaks are given in Table 2.

Particle size calculation from X-ray diffraction: Average particle size has been estimated by using Scherrer equation:

$$
\begin{aligned}
& \mathrm{D}=0.9 \lambda / \beta \cos \theta \\
& \mathrm{D}=34.75 \mathrm{~nm}
\end{aligned}
$$

The crystallite size of the samples increases with increase in sintering temperature. It is due to the increase in density with increasing sintering temperature (Islam et al., 2012).

\section{The lattice constant ' $a$ ' was calculated using the relation:}

$$
\begin{aligned}
& \mathrm{a}=\mathrm{d}_{\mathrm{hkl}} \sqrt{\mathrm{h}^{2}+\mathrm{k}^{2}+\mathrm{1}^{2}} \\
& \mathrm{a}=8.81 \mathrm{~nm}
\end{aligned}
$$

where, $h, k, 1$ are the Miller indices of the crystal planes and $d_{k k l}$ is the inter planer spacing. The lattice constant obtained from XRD data is in reported range.

\section{The $X$-ray density $\left(d_{x}\right)$ was calculated using the relation:}

$$
\begin{aligned}
& \mathrm{d}_{\mathrm{x}}=\frac{8 \mathrm{M}}{\mathrm{Na}^{3}} \\
& \mathrm{D}_{\mathrm{x}}=5.42 \mathrm{~g} / \mathrm{cm}^{3}
\end{aligned}
$$

Where:

$\mathrm{M}=$ Molecular weight

$\mathrm{N}=$ Avogadro's number

$\mathrm{a}=$ The Lattice constant

The X-ray density is found to be of the order of $5.42 \mathrm{~g} / \mathrm{cm}^{3}$. The bulk density of cobalt ferrite nano particles was measured from the Archimedes principle. The value of bulk density is of the order of $3.594 \mathrm{~g} / \mathrm{cm}^{3}$. The apparent density calculated by Archimedes method (Actual Density (D)) and the calculation of porosity using the equation:

$$
\begin{aligned}
& \text { Porosity }=[1-(\mathrm{D} / \mathrm{DX})]^{*} 100 \% \\
& \text { Porosity } \%=37.3
\end{aligned}
$$


(a)

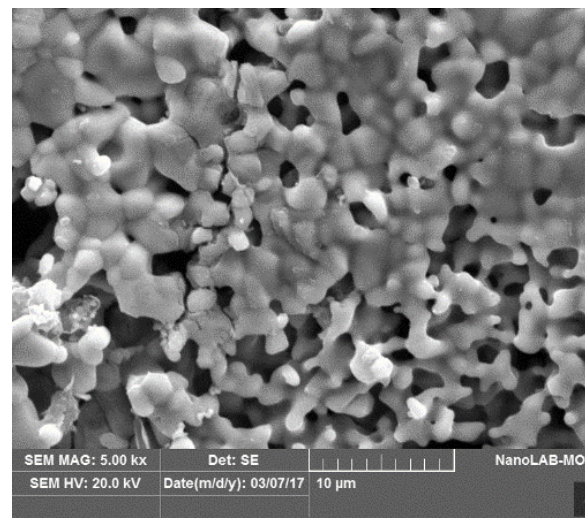

(b)

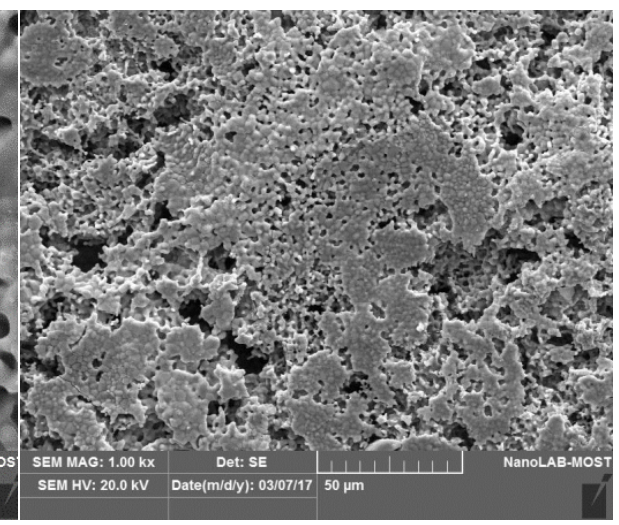

Fig. 3a, b): SEM images of cadmium ferrite sinteredat $1000^{\circ} \mathrm{C}$

Scanning electron microscope of cadmium ferrite: SEM measurements are carried out in order to understand the morphology and the shape of the synthesized nanomaterials. Scanning Electron Microscope (SEM) carried out on samples sintered at $1000^{\circ} \mathrm{C}$ was used to determine the lump size as shown in Fig. 3. From the micrograph, we can observe the formation of soft agglomerates with irregular morphology constituted the quite fine particles. Since, the ceramic method involves sintering of the stoichiometric mixtures at high temperatures, the crystallites are in order of micrometer with relatively smooth surface. The aggregate of crystallites of various sizes indicating a size distribution in the micrographs. These micrographs shows clearly that the network formation and agglomeration of the cadmium ferrite sample has taken place. SEM micrograph of cadmium ferrite pellet show the grain boundary of the sample being sintering at $1000^{\circ} \mathrm{C}$.

\section{CONCLUSION}

Ferrite samples under investigation show single phase cubic spinel structure. Porosity is found to decrease while slightly. The Lattice constant and grain size increase with increasing sintering temperature. The crystallite size of the samples lies in the range $34.75-34.75 \mathrm{~nm}$ an attempt was made to synthesize cadmium ferrite by a simple combustion method and was found to be very much suitable for obtaining cubic cadmium ferrite spinel.

\section{RECOMMENDATION}

Further study is being extended towards the synthesis and characterization of substituted rare-Earth elements by various techniques.

\section{REFERENCES}

Ahmed, M.A., N. Okasha and S.I. El-Dek, 2008. Preparation and characterization of nanometric $\mathrm{mn}$ ferrite via different methods. Nanotechnol., 19: 1-6.

Carter, C.B. and M.G. Norton, 2007. Ceramic Materials: Science and Engineering. Springer, Berlin, Germany, Pages: 691.

Chatterjee, A., D. Das, S.K. Pradhan and D. Chakravorty, 1993. Synthesis of nanocrystalline nickel-zinc ferrite by the sol-gel method. J. Magnetism Magn. Mater., 127: 214-218.

Cullity, B.D. and S.R. Stock, 2001. Elements of X-ray Diffraction. 3rdEdn., Prentice Hall, New Jersey, ISBN: 0201610914 , Pages: 664.

Gadkari, A.B., T.J. Shinde and P.N. Vasambekar, 2009. Structural analysis of $\mathrm{Sm} 3+$ doped nanocrystalline $\mathrm{Mg}$-Cd ferrites prepared by oxalate co-precipitation method. Mater. Charact., 60: 1328-1333.

Hall, B.D., D. Zanchet and D. Ugarte, 2000. Estimating nanoparticle size from diffraction measurements. J. Applied Crystallography, 33: 1335-1341.

Islam, R., M.O. Rahman, M.A. Hakim, D.K. Saha and S. Saiduzzaman et al., 2012. Effect of sintering temperature on structural and magnetic properties of Ni0.55Zn0.45Fe2O4 ferrites. Mater. Sci. Appl., 3: 326-331.

Mishra, S., N. Karak, T.K. Kundu, D. Das and N. Maity et al., 2006. Nanocrystalline nickel ferrites prepared by doping with niobium ions. Mater. Lett., 60: 1111-1115.

Mostafa, A.A., G.A. El-Shobaky and E. Girgis, 2006. Effects of $\mathrm{ZnO}$-Doping on structural and magnetic properties of $\mathrm{CdFe}_{2} \mathrm{O}_{4}$. J. Phys. D. Appl. Phys., 39: 2007-2014.

Nath, S.S., D. Chakdar and G. Gope, 2007. Synthesis of $\mathrm{CdS}$ and $\mathrm{ZnS}$ quantum dots and their applications in electronics. Nanotrends, 2: 1-5. 
Pradhan, S.K., S. Bid, M. Gateshki and V. Petkov, 2005. Microstructure characterization and cation distribution of nanocrystalline magnesium ferrite prepared by ball milling. Mater. Chem. Phys., 93: 224-230.

Suite, M., 1998. Soft ferrites: A user's guide. MBA Thesis, Magnetic Materials Producers Association, Mississauga, Ontario.
Vaingankar, A.S., S.G. Kulkarni and M.S. Sagare, 1997. Humidity sensing using soft ferrites. J. Phys., 7: 155-156.

Yamashiro, T., 1973. Electrical switching and momory phenomena in $\mathrm{CuFe}_{2} \mathrm{O}_{4}$. Japan. J. Appl. Phys., 12: 148-149.

Yokoyama, M., T. Sato, E. Ohta and T. Sato, 1996. Magnetization of cadmium ferrite prepared by coprecipitation. J. Appl. Phys., 80: 1015-1019. 